\title{
Facile Preparation of Alkylidenesuccinimides from Maleimides via the Phosphoniosilylation Process
}

\author{
Sun Ho Jung," Jung Hyun Kim, and Jung Hyun Lee \\ Department of Chemistry, Sungshin Women's Liniversit, Seoul 136-742, Korea \\ Received March 23, 2004
}

Key Words : Phosphoniosilylation, $\beta$ - $\Lambda$ lkylidenesuccinimide, $\alpha, \beta$-Unsaturated imides

\begin{abstract}
$\beta$-Functionalization of $\alpha \beta$-unsaturated carbonyl compounds can be effected by a variety of methods, which generally involve either the organometallic conjugate addition procedures followed by enolate trapping and subsequent oxidation $^{1}$ or the dipole reversal process involving the conversion of $\alpha, \beta$-unsaturated carbonyl compounds into $\beta$ acylvinyl anion equivalents. However, the former procedures are sometimes inadequate and the requisite organocuprates are difficult to obtain, ${ }^{2}$ In the case of enones, very efficient and practical $\beta$-functionalization methods utilizing the phosphoniosilylation process, one of the latter processes. have been previously developed by Kozikowski, Kim and Lee..$^{3-5}$ Recently, we have reported that such procedure can also be employed to $\alpha, \beta$-unsaturated lactones and esters by effecting $\beta$-alkylation, $\beta$-hydroxyalkylation, $\beta$-conjugate addition of them, and $\beta$-sulfenylation (Scheme 1), As an extension of these studies we became interested in investigating the possibility of phosphoniosilylation-based $\beta$ functionalizations of $\alpha, \beta$-unsaturated imides, maleimdes. Herein, we now wish to report facile preparation of
\end{abstract}

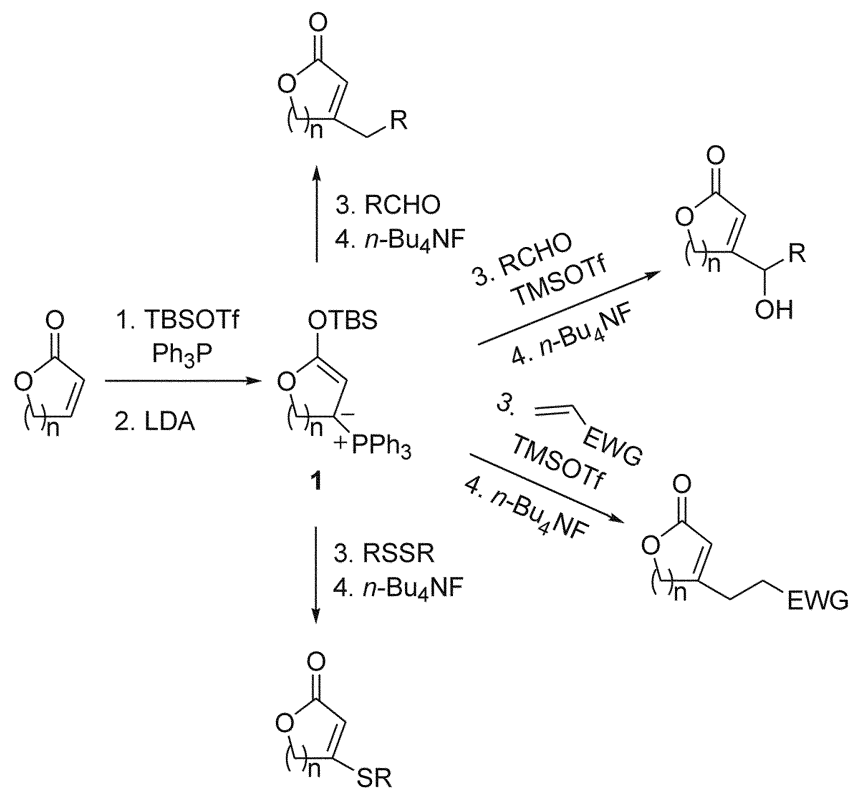

Scheme 1

Corresponding Author. Tel: -82-2-920-7192; Fax: +82-2-9293916; e-mail: shjung@sungshin.ac.kr alylidenesuccinimides from maleimides via the phosphoniosilylation process.

Since various $\beta$-functionalizations of enones, $\alpha, \beta$-unsaturated lactones and esters could be executed by utilizing the phosphoniosilylation process, ${ }^{3-6}$ we envisaged that alkylation of maleimides (2), might also be achieved. Thus, we examined the alkylation of maleimides. At the outset, scrutinizing the whole process of $\beta$-alkylation of $\alpha, \beta$-unsaturated lactones and esters, ${ }^{\text {(l) }}$ the feasibility of phosphoniosilylation and ylide formation was envisaged to be the key to success in alkylation of maleimides. Therefore, the facility of phosphoniosilylation and ylide formation was tested using $N$-methy] maleimide (2a) as a model compound. The phosphoniosilylation of $N$-methyl maleimide (2a) with triphenylphosphine and /-butyldimethylsilyl triflate (TBSOTf) proceeded rather favorably, and the resulting phosphonium salt also underwent smooth deprotonation by lithium diisopropylamide (I.DA) to give the ylide (3a). With success in these two steps, next, it was examined that the ylide (3a) would participate in the Wittig reaction and the following protodesilylation process in a desired sense. When the Wittig reagent (3a) was reacted with benzaldehyde at $-78^{\circ} \mathrm{C}$ to r.t. and then the mixture was treated with tetra- $n$-butylammonium fluoride (TBAF) in the same pot, $N$-methyl-3benzylidenesuccinimide (5a) was obtained as a major product along with small amount of benzylated maleimide (6a) in $43 \%$ yield. This result was rather unexpected in that $\alpha$-protonation was preferred in contrast to the preference of $\gamma$-protonation observed in cases of enones, $\alpha, \beta$-unsaturated lactones and esters. ${ }^{\text {iat } 6 \mathrm{~b}}$ Regardless of this result. we next tested the possibility of hydroxyalkylation of $N$-methyl maleimide $(\mathbf{2} \mathbf{a})$ by employing $\beta$-hydroxyalkylation procedure of $\alpha \beta$-unsaturated lactones and esters, ${ }^{\text {ob }}$ in which betaine intermediates were quenched by TMSOIf prior to the formation of oxaphosphetanes during the Wittig reaction. Thus, when the ylide ( $\mathbf{3} \mathbf{a})$ was reacted with benzaldehyde and TMSOlf at $-78^{\circ} \mathrm{C}$ for $0.5 \mathrm{~h}$, and the mixture was then treated with ' JBAF at $-78^{\circ} \mathrm{C}$ to rt. surprisingly, $N$-methyl-3benzylidenesuccinimide (5a) yielded as a major product rather than the desired hydroxybenzylated $N$-methylmaleimide (7a). The yield (68\%) of $N$-methyl-3-benzylidenesuccinimide (5a) obtained in this way was Imuch better (vide supro and entry 1, Jable 1). This result indicates that in the Wittig reaction of ylide (3a) with benzaldehyde, for some reason. TMSO'f plays as an activating agent to facilitate the 
<smiles>[R]N1C(=O)C=CC1=O</smiles>

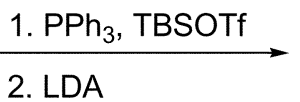

2

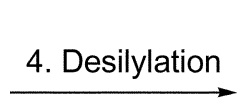<smiles>[R]C=C1CC(=O)N([R])C1=O</smiles>

5<smiles>[R17]N1C(=O)C(=P[SbH2])C=C1[O+]</smiles>

3<smiles>[R]C=C1C=C([OH+][BH3-])N([R1])C1=O</smiles>

4

for 2-3, $\begin{aligned} & a: R^{1}=\mathrm{CH}_{3} \\ & \text { b: } \mathrm{R}^{1}=\mathrm{Ph}\end{aligned}$

for 4-7,

a: $\mathrm{R}^{1}=\mathrm{CH}_{3}, \mathrm{R}^{2}=\mathrm{Ph}$

b: $\mathrm{R}^{1}=\mathrm{CH}_{3}, \mathrm{R}^{2}=p-\mathrm{CH}_{3} \mathrm{C}_{6} \mathrm{H}_{4}$

c: $\mathrm{R}^{1}=\mathrm{CH}_{3}, \mathrm{R}^{2}=p-\mathrm{CH}_{3} \mathrm{OC}_{6} \mathrm{H}_{4}$

d: $\mathrm{R}^{1}=\mathrm{CH}_{3}, \mathrm{R}^{2}=p-\mathrm{ClC}_{6} \mathrm{H}_{4}$

e: $\mathrm{R}^{1}=\mathrm{CH}_{3}, \mathrm{R}^{2}=\left(\mathrm{CH}_{2}\right)_{7} \mathrm{CH}_{3}$

f: $\mathrm{R}^{1}=\mathrm{CH}_{3}, \mathrm{R}^{2}=\left(\mathrm{CH}_{2}\right)_{2} \mathrm{CH}_{3}$<smiles>[R]C(O)C1=CC(=O)N([R])C1=O</smiles>

Scheme 2

attack of ylide (3a) on the carbonyl group rather than as a trapping agent to quench the betaine intermediate. This phenomenon was general in maleimide series. Therefore, the latter four-step one pot procedure was employed to prepare alkylidenesuccinimides from maleimides.

The results are shown in lable 1. This process works well with most aromatic and aliphatic aldehydes in $N$-methylmaleimide series (2a) in which products were obtained in moderate to good yields (51-85\%, entries 1-6). Similarly, with most aromatic and aliphatic aldehydes in $N$-phenylmaleimide series (2b), products were obtained in moderate yields $(46-56 \%$, entries $8-11)$. However, with isobutyraldehyde in both series, the yields which attend this procedure were relatively low (entries 7, 12). The low yields were assumed to be due to the bulky nature of isopropyl group.

On all occasions, alkylidenesuccinimides (5) were obtained as major products along with small amounts of alkylated maleimides (6). The ratios of 5 to $\mathbf{6}$ were $>10: \mathrm{I}$. The stereochemistry of alkylidenesuccinimides (5) was assigned as shown in 5, based on comparisons of ' $\mathrm{H}$ NMR data of 5 to those of authetic samples. ${ }^{7}$ The preferred formation of 5 to 6 seems to be attributed to kinetic favor of $\alpha$-protonation over $\gamma$ protonation in these cases. Thermodynamically, at least in some cases, alkylated maleimides (6) are more stable than alkylidenesuccinimides (5) as evidenced in successful isomerization of alkylidenesuccinimides (5a and $\mathbf{5 b}$ ) to alkylated maleimides ( $6 \mathbf{a}$ and $\mathbf{6 b}$ ) by treating DBU in refluxing THF (or toluene). Thus, although the isomerization requires long reaction time $(\sim 7$ days), one can also achieve alkylation of maleimides through isomerization following four-step one pot sequence of phophoniosilylation, phosphorane formation, Wittig reaction and protodesilylation.

The results illustrate the facility and applicability of the
Table 1. Preparation of $A \mathrm{ky}$ lidenesuecinimides from Maleimides

\begin{tabular}{|c|c|c|c|c|}
\hline entry & lmide & Aldchydc & Product & Yicld $(\%)$ \\
\hline & 2 & $\mathrm{R}^{2} \mathrm{CHO}$ & 5 & \\
\hline I & $2 a$ & $\mathrm{R}^{2}=\mathrm{Ph}$ & 5a & 68 \\
\hline 2 & $2 a$ & $\mathrm{R}^{2}=p-\mathrm{CH}_{3} \mathrm{C}_{6} \mathrm{H}_{4}$ & $5 \mathbf{b}$ & 64 \\
\hline 3 & $2 a$ & $\mathrm{R}^{2}=p-\mathrm{CH}_{3} \mathrm{OC}_{4} \mathrm{H}_{3}$ & $5 c$ & 51 \\
\hline 4 & $2 a$ & $\mathrm{R}^{2}=p-\mathrm{Cl}_{\mathrm{n}} \mathrm{H}_{1}$ & 5d & 85 \\
\hline 5 & $2 a$ & $\mathrm{R}^{2}=\left(\mathrm{CH}_{2}\right)_{7} \mathrm{CH}_{i}$ & $5 e$ & 79 \\
\hline 6 & $2 a$ & $\mathrm{R}^{2}=\left(\mathrm{CH}_{2}\right)_{2} \mathrm{CH}_{3}$ & $5 f$ & 66 \\
\hline 7 & $2 \mathrm{a}$ & $\mathrm{R}^{2}=\mathrm{CH}\left(\mathrm{CH}_{3}\right)_{2}$ & $5 g$ & 28 \\
\hline 8 & $2 b$ & $\mathrm{R}^{2}=\mathrm{Ph}$ & sh & $54^{\prime \prime}$ \\
\hline 9 & $2 b$ & $\mathrm{R}^{2}=p-\mathrm{CH}_{3} \mathrm{OC}_{4} \mathrm{H}_{3}$ & $5 i$ & $46^{\prime \prime}$ \\
\hline 10 & $2 b$ & $\mathrm{R}^{2}=\left(\mathrm{CH}_{2}\right)_{7} \mathrm{CH}_{3}$ & $5 j$ & 56 \\
\hline II & $2 b$ & $\mathrm{R}^{2}=\left(\mathrm{CH}_{2}\right)_{2} \mathrm{CH}_{3}$ & $5 \mathbf{k}$ & $55^{a}$ \\
\hline 12 & $2 b$ & $\mathrm{R}^{2}=\mathrm{CH}\left(\mathrm{CH}_{3}\right)_{2}$ & $\mathbf{5}$ & $22^{a i}$ \\
\hline
\end{tabular}

"salturated ammonium chloride solution was used instead of TBAF

present method. These overall conversions can be accomplished by four-step one pot procedure from maleimides without any isolation of the intermediates. Especially, it is noteworthy that in these cases TMSOTf plays as an activating agent to facilitate the attack of ylides on the carbonyl group rather than as a trapping agent to quench the betaine intermediates in the Wittig reaction.

In summary, we have shown that the phosphoniosilylation of maleimides, in combination with the Wittig reaction. provides a practical tool for the preparation of alkylidenesuccinimides from maleimides. One can also achieve alkylation of maleimdes by isomerization of alkylidenesuccinimides. 


\section{Experimental Section}

The general procedure for alkylidenesuccinimides from maleimides. To a solution of triphenylphosphine (146 mg. $0.55 \mathrm{~mm}$ ol) and $N$-methylmaleimide $(57.3 \mathrm{mg}, 0.50$ mmol ) in tetrahydrofuran $(2.0 \mathrm{~mL})$ was added TBSOTf (126 $\mu \mathrm{L} .0 .55 \mathrm{~mm}$ ol). After being stirred at room temperature for $1.5 \mathrm{~h}$. the reaction mixture was cooled to $-78^{\circ} \mathrm{C}$ and LDA. prepared from diisopropylamine ( $109 \mu \mathrm{L}, 0.78 \mathrm{mmol})$ and $n$ butyllithiun ( $4+2 \mu \mathrm{L}$ of $1.47 M$ solution in hexanes, 0.65 mmol) in THF, was added dropwise to give a dark browncolored solution. The mixture was stirred at $-78^{\circ} \mathrm{C}$ for $\mathrm{l}$ h and benzaldehyde $(66.1 \mu \mathrm{L}, 0.65 \mathrm{mmol})$ was added prior to the quick addition of TMSOTf (136 $\mu \mathrm{L}, 0.75 \mathrm{mmol})$. After the reaction mixture was stirred for $0.5 \mathrm{~h}$. TBAF $(2.0 \mathrm{~mL}$ of $1 M$ solution in THF, $2.0 \mathrm{mmol}$ ) was added. After being warmed to room temperature. the reaction mixture was stirred for $1.5 \mathrm{~h}$. The extractive work-up and flash column chrontatography gave $N$-methyl-3-benzylidenesuccinimide (5a. entry l) $(68.1 \mathrm{mg} .68 \%) .{ }^{1} \mathrm{H} \mathrm{NMR}\left(500 \mathrm{MHz}^{\left.-\mathrm{CDCl}_{\mathfrak{j}}\right) \delta}\right.$ 7.64 (s. $1 \mathrm{H}), 7.52-7.4 \mathrm{l}$ (n. $5 \mathrm{H}) .3 .59$ (s. $2 \mathrm{H}), 3.14(\mathrm{~s}, 3 \mathrm{H})$. ${ }^{13} \mathrm{C} \mathrm{NMR}\left(125 \mathrm{MHz}, \mathrm{CDCl}_{3}\right) \delta 174.1,171.1 .134 .3,134.1$. 130.2, 130.1. 129.1. 123.5, 34.1. 24.9. IR (KBr) 2945. 1706. 1655, 1434, 1378, 1281. $784 \mathrm{~cm}^{-1}$.

N-Methyl-3-(4-methylbenzylidene)succinimide (5b, entry 2). ${ }^{3} \mathrm{H}$ NMR (200 MHz. $\left.\mathrm{CDCl}_{3}\right) \delta 7.62$ (s, IH). 7.41 $(\mathrm{d}, j=7.81 \mathrm{~Hz} .2 \mathrm{H}) .7 .28(\mathrm{~d} . J=7.81 \mathrm{~Hz} .2 \mathrm{H}), 3.58(\mathrm{~s}, 2 \mathrm{H})$. 3.15 (s, 3H). 2.42 (s. 3H). IR (KBr) 2950. 1706. 1655.1440. $1383,1281,820.743(=\mathrm{CH}) \mathrm{cm}^{-1}$.

$\mathrm{N}$-Methyl-3-(4-methoxylbenzylidene)succinimide (5c, entry 3). ${ }^{j} \mathrm{H}$ NMR $\left(200 \mathrm{MHz} . \mathrm{CDCl}_{3}\right) \delta 7.60$ (s, IH). 7.48 $(\mathrm{d}, J=8.30 \mathrm{~Hz} .2 \mathrm{H}$ ). 6.95 (d. $J=8.79 \mathrm{~Hz} .2 \mathrm{H}), 3.88(\mathrm{~s}, 3 \mathrm{H}$ ). $3.57(\mathrm{~s}, 2 \mathrm{H}) .3 .14$ (s. $3 \mathrm{H})$. IR (KBr) 2935. 1706. 1655.1434. $1388,1281,835.748 \mathrm{~cm}^{-1}$.

$N$-Methyl-3-(4-Chlorobenzylidene)succinimide $\mathbf{( 5 d}$, entry 4). ${ }^{1} \mathrm{H}$ NMR $\left(500 \mathrm{MHz} . \mathrm{CDCl}_{3}\right) \delta 7.56$ (s, IH). 7.437.39 (s. $4 \mathrm{H}), 3.53$ (s. $2 \mathrm{H}) .3 .12(\mathrm{~s}, 3 \mathrm{H}) .{ }^{13} \mathrm{C}$ NMR $(125 \mathrm{MHz}$. $\left.\mathrm{CDCl}_{3}\right) \delta 173.7,170.9,136.3,132.9,132.6 .131 .2 .129 .5$. 124.1, 33.9. 25.0. IR (KBr) 2941, 1701, 1654, 1437. 1386. $1278,1025,706,554 \mathrm{~cm}^{-1}$

$N$-Methyl-3-nonylidenesuccinimide (5e, entry 5). ${ }^{\text {}} \mathrm{H}$ NMR $\left(200 \mathrm{MHz}^{\circ} \mathrm{CDCl}_{3}\right) \delta 6.84(\mathrm{t} . J=7.33 \mathrm{~Hz}, 1 \mathrm{H}), 3.22$ (s. $2 \mathrm{H}) .3 .08$ (s. 3H) 2.19 (q. $J=7.33 \mathrm{~Hz}, 2 \mathrm{H}), 1.29$ (s. 12H). $0.89(\mathrm{t} . J=6.10 \mathrm{~Hz}, 3 \mathrm{H})$. IR (KBr) $2935,2925,2853.1706$. 1660.1440,1388,1281.743 $\mathrm{cm}^{-1}$.

$N$-Methyl-3-butylidenesuccinimide (5f, entry 6). ${ }^{1} \mathrm{H}$ NMR $\left(200 \mathrm{MHz}^{\circ} \mathrm{CDCl}_{\mathrm{j}}\right) \delta 6.85$ (t. $\left.\mathrm{lH}\right) .3 .23(\mathrm{~s} .2 \mathrm{H}) .3 .09$ (s. $3 \mathrm{H}) .2 .20(\mathrm{q} . J=7.33 \mathrm{~Hz}, 2 \mathrm{H}) .1 .66-1.47(\mathrm{~m}, 2 \mathrm{H}) .0 .99(\mathrm{t}$. $J=7.33 \mathrm{~Hz}, 3 \mathrm{H})$. IR (KBr) 2966, 2935. 2868, 1706, 1660 . 1445, 1388, 1281, $743 \mathrm{~cm}^{-1}$.

$N$-Methyl-3-isobutylidenesuccinimide (5g, entry 7). ${ }^{1} \mathrm{H}$ NMR $\left(200 \mathrm{MHz}^{\circ} \mathrm{CDCl}_{j}\right) \delta 6.68(\mathrm{~d}, J=9.77 \mathrm{~Hz}, 1 \mathrm{H}) .3 .24$ (s. 2H). 3.07 (s. 3H) . 2.54-2.43 (m. 1H). 1.10 (d. $J=6.84 \mathrm{~Hz}$. $6 \mathrm{H})$. IR (KBr) 2966, 2930, 2873, 1706, 1660, 1445, 1388. 1281, $738 \mathrm{~cm}^{-1}$.

$N$-Phenyl-3-benzylidenesuccinimide (5h, entry 8). ${ }^{\text {}} \mathrm{H}$
$\operatorname{NMR}\left(200 \mathrm{MHz}, \mathrm{CDCl}_{3}\right) \delta 7.77(\mathrm{~s}, \mathrm{lH}) .7 .55-7.38(\mathrm{~m}, \mathrm{IOH})$. $3.79(\mathrm{~s}, 2 \mathrm{H})$. IR (KBr) 3058. 2945, 1710. 1650, 1501. 1393. 1281. $743 \mathrm{~cm}^{-1}$

$\mathrm{N}$-Phenyl-3-(4-methoxybenzylidene)succinimide (5i, entry 9). ${ }^{\mathrm{H}} \mathrm{H} \mathrm{NMR}\left(200 \mathrm{MHz}, \mathrm{CDCl}_{3}\right) \delta 7.72(\mathrm{~s}, \mathrm{lH}) .7 .55-$ 7.45 (m. $5 \mathrm{H}$ ). 7.40 (d. $J=6.35 \mathrm{~Hz} .2 \mathrm{H}) .7 .02$ (d. $J=8.79 \mathrm{~Hz}$ 2H). 3.90 (s, 3H). 3.76 (s. 2H). IR (KBr) 3058. 2966, 1706, 1655. 1511. 1388. 1265, 846.702 $\mathrm{cm}^{-1}$.

$N$-Phenyl-3-nonylidenesuccinimide (5j, entry 10). ${ }^{\mathrm{l}} \mathrm{H}$ NMR $\left(200 \mathrm{MHz} . \mathrm{CDCl}_{3}\right) \delta 7.54-7.33(\mathrm{~m} .5 \mathrm{H}) .6 .97$ (t. $J=$ $7.33 \mathrm{~Hz} .1 \mathrm{H}$ ). $3.4 \mathrm{l}$ (s. $2 \mathrm{H}$ ). 2.26 (q. $J=7.33 \mathrm{~Hz}, 2 \mathrm{H}$ ), 1.30 (s. $12 \mathrm{H}), 0.91$ (t. $J=6.10 \mathrm{~Hz}, 3 \mathrm{H})$. IR (KBr) 2966. 2930,2853 , 1711. 1665. 1516. 1388, $1250,712 \mathrm{~cm}^{-1}$

$N$-Phenyl-3-butylidenesuccinimide (5k, entry 11). ${ }^{1} \mathrm{H}$ NMR $\left(200 \mathrm{MHz} . \mathrm{CDCl}_{3}\right) \delta 7.54-7.32(\mathrm{~m} .5 \mathrm{H}) .6 .98(\mathrm{t} . J=$ $7.81 \mathrm{~Hz}, \mathrm{lH}) .3 .42$ (s, 2H). $2.26(\mathrm{q}, J=7.33 \mathrm{~Hz}, 2 \mathrm{H}) .1 .69-$ $1.5 \mathrm{l}$ (m. 2H). 1.02 (t. $J=7.33 \mathrm{~Hz} .3 \mathrm{H}$ ). IR (KBr) 2970 , 2940. 1706. 1650.1506, 1388, 1255,746 $\mathrm{cm}^{-1}$

$\mathrm{N}$-Phenyl-3-isobutylidenesuccinimide (5l, entry 12). ${ }^{\mathrm{H}} \mathrm{H}$ NMR $\left(200 \mathrm{MHz}, \mathrm{CDCl}_{3}\right) \delta 7.42(\mathrm{~m}, 5 \mathrm{H}) .6 .81(\mathrm{~d} . J=6.84$ Hz. IH). 3.43 (s, 2H). 2.58-2.55 (m. lH). 1.14 (d. $J=6.84$ Hz. 6H). IR (KBr) 2966. 2930. 2863. 1711, 1650, 15l1, 1388. $1250.738 \mathrm{~cm}^{-1}$.

3-Benzyl-1-methyl-pyrrole-2,5-dione (6a). ${ }^{1} \mathrm{H}$ NMR $(500$ $\mathrm{MHz}, \mathrm{CDCl}_{3}$ ) $\delta 7.35-7.27$ (m. 4H). 7.07 (d, 2H). 2.94 (s. 3H). $2.78(\mathrm{~s}, 2 \mathrm{H}) .{ }^{12} \mathrm{C} \mathrm{NMR}\left(125 \mathrm{MHz} . \mathrm{CDCl}_{3}\right) \delta 173.9$ (2C), 135.8, 130.1. 129.0. 128.9, 128.0, 126.2, 38.2, 24.5. IR (KBr) $2927.1702 .1690,1438,1388.1285 .768 \mathrm{~cm}^{-1}$.

Acknowledgement. This work was supported by a Grant from Sungshin Women's University in 2003.

\section{References}

1. (a) Posner. G. H. An Introduction to Stmthesis Lsing Organocopper Reagunts; Wiley-Interscience: New York. 1980. (b) Taylor. R. J. K. Organocopper Reagents: Oxford University Press: Dxford. 1994

2. (a) Taylor. R. T. K. Symthesis 1985. 364. (b) Perlmutter. P. Contugate Addition Reactions in Onganic Swmthesis. Pergamon Press: Oxtord, 1992. (c) Hulce, M. Org. React. 1990. 38,225. (d) Lipshutz, B. H.: Sengupta. S. Org. React. 1992, H. 135.

3. (a) Kozitowshi, A. P: Jung, S. H. d. Ong Chem. 1986, 51,3400 (b) Kozikowski. A. P.: Jung. S. H. Tetrahedron Lett. 1986. 27.3227.

4. (a) Kim. S.: Lee. P. H. Tetrahedon Lett. 1988. 29. 5413. (b) Kim. S.: Lee. P. H.: Kim. S. S.: Bull. Korean Chem. Soc. 1989. 10. 218 (c) Kim, S.: Kim, Y. G.: Park. J. H. Tetrahedon Let, 1991, 32. 2043. (d) Kim. S: Park. J. H: Kim. Y. G.: Lee, J. M d. Chem. Soc., Chem. Commm. 1993. 1188. (e) Kim, S.; Lee, B. S.: Park, J H. Bull. Korean Chem. Soc. 1993. 14. 654.

5. (a) Lee. P. H: Kim. S. Bull. Korean Chem. Soc. 1992. 13. 580 . (b) Lee. P. H.: Cho. M:: Hatı. I.-S.: Kim. S. Tetrahedron Lett. 1999. to. 6975 .

6. (a) Jung. S. H.; Kim. J. H. Bull. Konam Chem. Soc. 2002. 23,365 (b) Jung. S. H. Kim, J. H. Bull. Korean Chem. Soc. 2002, 23. 1375. (c) Jung. S. H.: Kiml. T. H.: Kiml. H. T. Bull. Korean Chem. Soc. 2004. 24. 136.

7. (a) Paternotte. I.: Fan. H. I.: Screve. P.: Cllaesen. M.: Tulkens. P. M. Sonveaux. E. Bioorg. Med Chent 2001, 9. 493. (b) Hedaya. E:: Theodoropulos, S. Tetralkadron 1968, 24, 2241 